\title{
A Reputation Evaluation Approach Based on Fuzzy Relation
}

\author{
Meiyu Fang ${ }^{1}$, Xiaolin Zheng ${ }^{2}$ (corresponding author), Deren Chen ${ }^{3}$ \\ ${ }^{1}$ College of Computer Science, Zhejiang University ${ }^{(a)}$, \\ College of Computer Science, Zhejiang International Study University ${ }^{(b)}$, \\ Hangzhou, China \\ Email:hwdfmy@yahoo.com.cn \\ ${ }^{2}$ College of Computer Science, Zhejiang University, \\ Hangzhou, China \\ Email:xlzheng@cs.zju.edu.cn \\ ${ }^{3}$ College of Computer Science, Zhejiang University, \\ Hangzhou, China \\ Email:drc@zju.edu.cn
}

\begin{abstract}
In traditional models, fuzzy sets are used to describe trust degree and evaluate reputation for vague words. But in some practical applications, the determination of membership functions associated with vague concepts is difficult or impossible. This paper builds a reputation computing model based on fuzzy relation and provides a new reputation evaluation approach among vague concepts. The definition of reputation degree computing is given according trust passing and trust combination. The experimental results show the effectiveness of the proposed approach in reputation comparison among different sellers and various index assessment among products of one seller.
\end{abstract}

Keywords: reputation evaluation; fuzzy relation; reputation computing;trust passing; trust combination.

\section{Introduction}

In the open web environments, electronic commerce has been developing quickly; reputation research is becoming increasingly more urgent. Trust degree and reputation may directly impact an agent's transaction decision-making[1].Although the rating grade can provide some reputation information available[2], many experienced net buyers would like to evaluate online stores' reputation with vague and imprecise reviews written by other online shoppers but not the rating grade[3].Fuzzy nature is an important and the most complicated feature of trust, it makes the trust measurement very difficult.

Fuzzy relation was proposed by Yongchuan Tang for modeling linguistic labels in literature [4].We extended fuzzy relation to a reputation model called RMBFR. Then a reputation evaluation approach was designed based on RMBFR. The process of this method's algorithm adopted an epistemic view since the input and the output are consistent with vague words.Because the subjective trust information collected from individual agent are often represented by a set of vague linguistic words, the uncertain rea- 
soning are involved for trust or reputation computing.This model can well overcome the specific information deletion in traditional model which adopted the numeric rate matrix or the subjective empirical fuzzy membership function.

The paper is structured as follows: section 2 describes some related work about the situation of probability and fuzzy set. Section 3 elaborates the theoretic foundation, Tang's fuzzy relation. Section 4 shows the proposed model and section 5 demonstrates the model's algorithm of section 4. Section 6 shows the experimental results of reputation comparison and in section 7, conclusion and future work are presented. The last section is our acknowledgements.

\section{Related works}

In classical reputation modeling $[5,8]$,one representative model was proposed by JØsang who adopted subjective logic and introduced evidence space and opinion space to describe and measure trust relation $[5,6,7]$. Based on the Beth distribution function for describing the binomial-event, Jりsang's model determined the probability density function according to the numbers of the certain events and the negative events. Jりsang reflected the trust subjectivity and uncertainty to the probability; it was unavoidable to lead the subject relief to randomness.

The other types of representative models are most coming from Zadeh's fuzzy set theory or the extension of it[9]. Among these models, some of them processed the vague number information[11,12]which may lead to inefficiency in practical applications, the others computed the information with vague words[10]. Zadeh firstly introduced the concept of linguistic variable as a model of how words or labels can represent vague concepts in natural language [8]. The use of fuzzy sets is central to computing with words as they provide a means of model vagueness underlying most natural language terms [10]. But, in some practical applications, the determination of membership functions or fuzzy sets associated with linguistic labels is difficult or impossible[12].

Some linguistic labels, such as grand and steady, are often applied to product design evaluation. In fact, the linguistic uncertainty can be directly modeled by a fuzzy relation on the set of linguistic words. This fuzzy relation can be interpreted as the degrees of semantic similarities among linguistic labels. This paper uses fuzzy relation to describe the degrees of similarities among user comment matrices, instead of fuzzy sets to describe the semantics of words. The main contribution of this paper is to directly build the reputation model RMBFR based on fuzzy relation among vague comment words, but not fuzzy sets computing with words, neither trust matrices using the rate grade numbers.

\section{Reputation Model Based on Fuzzy Relation}

We extended fuzzy relation to a reputation model called RMBFR. The process of RMBFR adopts an epistemic view since the input and the output are consistent with vague words.Because the subjective trust information collected from individual agent is often represented by a set of vague linguistic words, the uncertain reasoning was involved in trust computing. A new trust passing computing approach and a trust combination method are proposed in this paper. RMBFR establishes another computing principle of linguistic label which is different from fuzzy set theory, and provide basis for the subsequent algorithm.

\subsection{Fuzzy Relation(FR)}

Tang's fuzzy relation[4] concentrates on a fuzzy relation $\mathrm{R}=\left(r_{i j}\right)_{n \times n}$ on an underlying finite set of linguistic labels $\mathrm{LA}=L_{i}, \ldots, L_{n}$. where the element $r_{i j} \in[0,1]$ in the ith row and jth column of $\mathrm{R}$ represents the degree of semantic overlapping or similarity between linguistic labels $L_{i}$ and $L_{j}$, or 1 . $r_{i j}$ represents the semantic difference between linguistic labels $L_{i}$ and $L_{j}$. Tang's FR showed that the logic operators ' $\wedge$ ' and ' $V$ ' are consistent with the t-norm operation 'min' and t-norm operation 'max' in the fuzzy logic and hold the middle law and the non-contradiction in two valued logic. In this paper, we use LA as the users' estimation scale set, $\mathrm{R}$ as relation set on LA. That is to say, each users' assessment index is divide into many vague grade 
expressed by linguistic label in LA. R matrix elements represent the similarity between any two labels.Tang's FR supports the computing as follows:

1) The Definition of Linguistic expression LE: $L_{i} \in$ LE for $\mathrm{i}=1, \ldots, \mathrm{n}$;if $\theta, \phi \in \mathrm{LE}$, then

$$
\neg \theta, \theta \wedge \phi, \theta \vee \phi, \theta \rightarrow \phi \in L E .
$$

2)If $L_{i_{k}} \in \mathrm{LA}$ for $\mathrm{k}=1, \ldots, \mathrm{m}$ and $\theta \in \mathrm{LE}$ then

$$
r\left(L_{i_{1}} \wedge L_{i_{2}} \wedge \ldots \wedge L_{i_{m}}, \theta\right)=\min _{k=1, \ldots, m} r\left(L_{i_{k}}, \theta\right) .
$$

3)If $L_{i_{k}} \in \mathrm{LA}$ for $\mathrm{k}=1, \ldots, \mathrm{m}$ and $\theta \in \mathrm{LE}$ then

$$
r\left(L_{i_{1}} \vee L_{i_{2}} \vee \ldots \vee L_{i_{m}}, \theta\right)=\max _{k=1, \ldots, m} r\left(L_{i_{k}}, \theta\right) .
$$

4)For any $\theta, \phi \in \mathrm{LE}$, the following formulas hold,

$$
\begin{aligned}
& r(\neg \theta, \phi)=1-r(\theta, \phi) \\
& r(\neg \theta \wedge \theta, \phi)=0 \\
& r(\neg \theta \vee \theta, \phi)=1 .
\end{aligned}
$$

\subsubsection{Reputation Definition}

The study and evaluation of reputation has attracted the interest of scientists from different fields such as: sociology [13], economics [13], psychology [15] and computer science [2,7,and 12]. According to the Oxford English Dictionary, reputation is the common or general estimate of a person or something with respect to character or other qualities etc. In literature [1], reputation is an aggregate value comes from the trust degree of all recommendation around the trust-get-agent, for example, the QoP or QoS [1] of the trust-get-agent. Of course, the details about the implications of trust degree, trust-get-agent,and trust-request-agent have been stated in the literature [1]. As we all know, in fact, the meaning of reputation is relative to its detail of background and the given time interval. In this paper, we define reputation in the $\mathrm{C} 2 \mathrm{C}$ e-commerce environments, and our view tends to agree with the definition in [1]. But we emphasize the value of the reputation aggregation and the associative concepts are vague concept or language, and our evaluation approach uses the fuzzy relation, but not the member function, to process the language. For example, trust degree computing adopts the vague comments evaluation and the relation between the element set but not the users' rate and the fuzzy set. In this paper, we call the trust aggregation from the history user as history reputation, and the final value from the historyreputation, the recommendation reputation as queryreputation.

\subsection{Building a Model RMBFR}

Reputation has some attributes we must obey such as subjectivity,fuzziness, asymmetry,transitivity,dynamic content relevance weakening with time, and reputation as a vector from reputation-query agent to reputation-get agent. According to those characteristics above, a reputation model is defined as a 5-tuple $<\mathrm{Ue}, \mathrm{La}, \mathrm{R}, \mathrm{T}(\mathrm{t}), \mathrm{B}(\theta)>$. In the 5-tuple, $\mathrm{Ue}$ is a users' evaluation matrix about some subject $\mathrm{X}, \mathrm{X}=x_{1}, x_{2}, \ldots, x_{n}$ is the reputation question area. $x_{i}$ represent one side of $\mathrm{X}$ such as $\mathrm{X}$ is a dress company, $x_{1}$ is the element expression of LA which review the price,$x_{2}$ the quality... La is equivalent to Tang's LA, it is a linguistic label set $L_{i}, \ldots, L_{n}$ which reflects the trust grade that a user assessed $x_{i}$ of a product of subject $\mathrm{X}$;for example, when $\mathrm{n}=5, \mathrm{La}=\{\mathrm{VB}$ (very bad), B(bad), N(normal), G(good), $\mathrm{VG}$ (very good)\}. According the epistemic view, a similarity exists between every two labels in LA(e.g. very bad and bad). One purchaser's comment for his or her transaction is "The price is very cheap,the quality is good,the appearance is very beautiful,but it is too large for me... ". So $\mathrm{x} 1=$ "VG"(The price is very cheap), $\mathrm{x} 2=" G$ " $\wedge$ "B" ("G" expresses the quality is good," $\mathrm{B}$ " points the bad aspect "it is large for me"). $T(t)$ indicates the evaluation TimeBucket of RMBFR, and $\mathrm{B}(\theta)$ indicates the background factor. For instance, we need to assess one $\mathrm{C} 2 \mathrm{C}$ enterprise one day's reputation. Then $\mathrm{t}$ points to the day and $\theta \mathrm{C} 2 \mathrm{C}$. So, the data in matrix Ue must have the time attribute which belongs to $t$. Because of the different scope of the transaction contents, we use different linguistic labels to divide the grade of the commodities. So $\mathrm{B}(\theta)$ is always expressed in one field vocabularies. For example, in the clothing industry, a common review may include a statement such as, "This dress is beautiful, very fitted, the material is very good, or it looks ugly, very large, the material is too bad." . In brief, the similarity 
degree between the linguistic labels is relative to $\mathrm{B}(\theta)$.In this paper, $\theta_{s}$ is a $\mathrm{B}(\theta)$ factor which is used to express the similarity degree between two neighboring labels when they are divide into several grades. $\mathrm{R}$ just like the $\mathrm{R}$ of Tang's fuzzy relation $\left(r_{i j}\right)_{n \times n} . \theta_{s}$ expresses the similarity degree between any two neighboring labels in La. Then we know that the difference degree between any two neighboring labels in $\mathrm{La}$ is $1-\theta_{s}$. We think that "very good" is much closer to "good" than "normal", and "good" is much closer to "normal" than "bad" and so on. Therefore, one possible definition of fuzzy relation $\mathrm{R}$ on La satisfies the relation of Fig1.

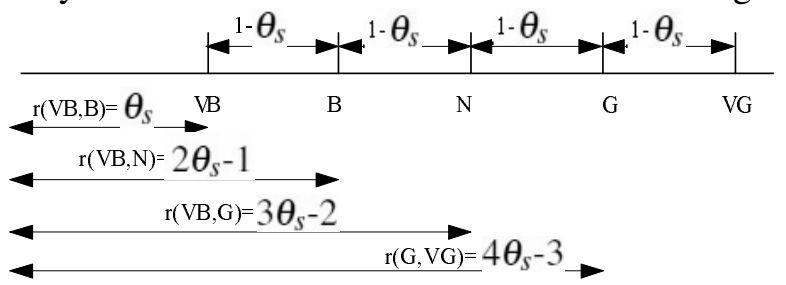

Fig. 1. The similarity relation of the label in La

From Fig1.we can see that the similarity degree of any adjacent label in $\mathrm{La}$ is $\theta_{s}$, and any similarity of itself equals 1 . Of course, the difference degree of any adjacent label is $1-\theta_{s}$. So for any $L_{i}, L_{i+1}, L_{j} \in \mathrm{La}, 0 \leqslant \mathrm{i}, \mathrm{j} \leqslant \mathrm{n}, \mathrm{r}\left(L_{i}, L_{i+1}\right)=\mathrm{r}\left(L_{i+1}, L_{i}\right)=\theta_{s}$, and $\mathrm{r}\left(L_{i}, L_{j}\right)=\mathrm{r}\left(L_{j}, L_{i}\right)=|i-j| \theta_{s}-(|i-j|-1)$, For example, $\quad r(\mathrm{VB}, \mathrm{B})=\mathrm{r}(\mathrm{B}, \mathrm{N})=\mathrm{r}(\mathrm{N}, \mathrm{G})=\mathrm{r}(\mathrm{G}, \mathrm{VG})=\mathrm{r}($ very good, good $)=\theta_{s}, \quad r(V G, N)=r(N, V G)=r($ very good, normal $)=2 \theta_{s}-1, \quad r(V G, B)=r(B, V G)=r($ very good, bad $)=3 \theta_{s}-2, \quad r(G, N)=r(N, G)=r(\operatorname{good}$, normal $)=\theta_{s}$, $\mathrm{r}(\mathrm{VG}, \mathrm{VB})=\mathrm{r}(\mathrm{VB}, \mathrm{VG})=\mathrm{r}($ very good, very bad $)=4 \theta_{s^{-}}$ 3. So, $\mathrm{R}$ matrix is derived.

$$
\boldsymbol{R}=\left[\begin{array}{ccc}
1 & \cdots & k \boldsymbol{\theta}_{s}-(k-1) \\
\vdots & |(i-j)| \boldsymbol{\theta}_{s}-|i-j|+1 & \vdots \\
k \theta_{s}-(k-1) & \cdots & 1
\end{array}\right]
$$

\section{Reputation Computing}

In the open net environment, Subject X's reputation can be described by a vector $\mathrm{Vr}$ on $\mathrm{La}$, $\mathrm{Vr}=\left\{v_{1}, v_{2}, \ldots, v_{n}\right\} . \quad v_{i}(\mathrm{i}=1,2, \ldots, \mathrm{i} \leqslant \mathrm{n})$ expresses the vague trust degree value of $x_{i}$ on $L_{i}$. For instance, a buyer's message is "This dress's appearance is very good, but the other ( for example the quality) is not good", So we can define that $v_{1}$ as the dress's appearance, $v_{1}=\mathrm{VG}$ (the value of $\left.L_{1}\right), v_{2}$ as the quality, $v_{2}=\neg \mathrm{VG}$, the logic expression of $\mathrm{VG}[2]$.

In general, in C2C E-business, a customer assesses the goods or the service after a successful transaction. According the way a person trusts something in natural, the more the customer appraises the commodities, and the greater he or she trusts the seller. So we define an ith user's trust degree as $U T_{i}$ for an agent(one seller)X through the evaluation $V_{k}$ about $x_{i}$ as follows:

$$
U T_{i}=\bigwedge_{k=1, \ldots, n} V_{k} \text { where } V_{k} \in V r .
$$

According the principle of reputation aggregation[1], history-reputation comes from all the trust degree $U T_{i}$ through the users' transaction history in time period $t$. Here the aggregation abides by the logic process as Tang's. So, the history-reputation about subject $\mathrm{X}$, expressed by h-REP is deducted:

$$
h-R E P=\frac{1}{n} \sum_{i=1, \ldots, n} U T_{i} .
$$

\subsection{Reputation Transitivity}

Suppose that $X_{j}$ is the reputation-query agent, $X_{i}$ is the third recommendation agent and $X_{k}$ is a reputation-gain agent, their relation is showed in Fig2. $\quad V_{1}$ is the reputation vector of $X_{i}$ that $X_{j}$ queried,$V_{2}$ is the reputation vector for the other agent $X_{k}$ which $X_{j}$ recommend to $X_{i}$. In Fig2., the reputation vectors have vector from the querying side to the gaining side.

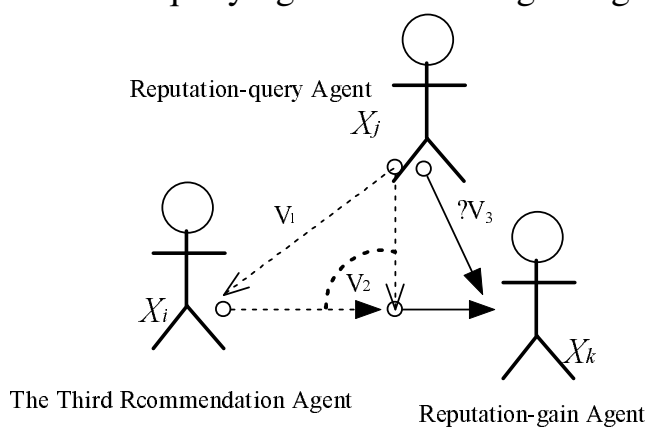

Fig. 2. The recommend relation of $X_{i}, X_{j}$ and $X_{k}$

In real life, we usually consider the recommender's reputation $V_{1}$ and its recommendation reputation $V_{2}$ to determination $V_{3}$. That is to say there 
exists a reasoning rule if the recommender's reputation is higher, the more the recommendation reputation will be accepted. So we defined the reputation transitivity computing as a connectivity computing:

$$
V_{3}=V_{1} \bigodot V_{2}=V_{1}^{T} \times V_{2}
$$

\subsection{Reputation Combination}

An agent's reputation is not only relative to its trust degree from all the buyers (see $V_{t}$ in Fig3) but is also relative to all the reputation coming from the direct query agent(see $V_{m}$ in Fig3) and the recommend reputation(see $V_{k}$ in Fig3). As Fig3 showing: $X_{k}$ 's reputation comes from $X_{i}, X_{j}, X_{m}, X_{n}, X_{t}$ etc. From this figure, we also determin that the reputation has a obvious dynamic feature because when the customers and its number transacted with the agent changes the reputation will not be the same. We define this reputation combination computing as the following:

$$
V_{\text {rep }}=V_{1} \bigoplus V_{2} \bigoplus \ldots \bigoplus V_{n}=\frac{1}{n} \sum_{i=1, \ldots, n} V_{i}
$$

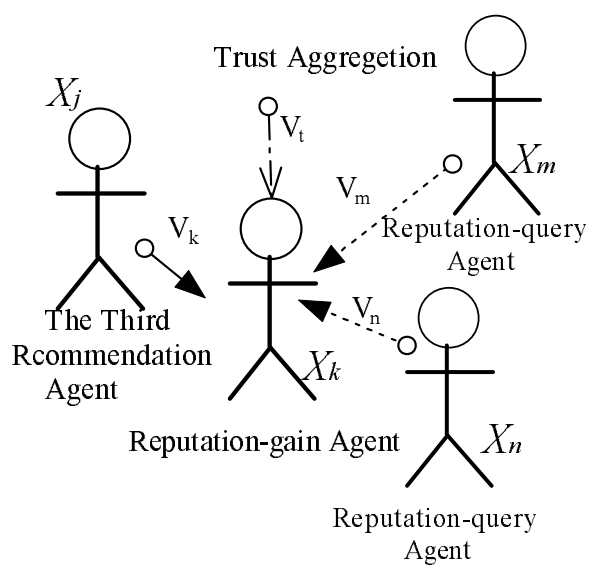

Fig. 3. The reputation combination

\section{Reputation Evaluating Algorithm}

In step 1, some data are initialized. La is assumed to 5 grade: $\mathrm{La}=\{$ "VB",'B",'N",',G",'VG" $\}$, then $\mathrm{La}_{0}=" \mathrm{VB} ", \mathrm{La}_{1}=" \mathrm{~B}$ " and so on. The user evaluation matrix are derived from random La labels' expression (LE : mentioned in Literature [4]). For distinguishing's sake, a different company's user review matrix randomly comes from the label set including different frequency elemental labels. If buyers have a bad impression on a company, more "B"s and "VB"s are included in the original label set. On the contrary, more "G"s and "VG"s are contained. In accordance in the natural, the more "good" and "very good" the buyers reviewed, the better the company's reputation would be. In light of equation matrix (7)in the preceding part of the paper, a relation matrix can be derived as following, $r[i][j]$ is an element of ith row and jth col in $\mathrm{R}$ :

$$
R=\left[\begin{array}{ccccc}
1 & \theta_{s} & 2 \theta_{s}-1 & 3 \theta_{s}-2 & 4 \theta_{s}-3 \\
\theta_{s} & 1 & \theta_{s} & 2 \theta_{s}-1 & 3 \theta_{s}-2 \\
2 \theta_{s}-1 & \theta_{s} & 1 & \theta_{s} & 2 \theta_{s}-1 \\
3 \theta_{s}-2 & 2 \theta_{s}-1 & \theta_{s} & 1 & \theta_{s} \\
4 \theta_{s}-3 & 3 \theta_{s}-2 & 2 \theta_{s}-1 & \theta_{s} & 1
\end{array}\right]
$$

In step 2, we design the structure of Ue as shown in table 1. Each row is a user evaluation vector $V_{r}$. Each $V_{r}$ consists of six items $V_{i}$ : pri(Is the price bad or good?), qua(Is the quality low-grade or excellent?), appe(Is the appearance beautiful or ugly,the size large or small?), ser(The service is satisfactory? ),mail(Is the mail fast or slow?) and time(The time property of $V_{r}$,it points the leaving message time).

Table 1. User Evaluation Data Structure.

\begin{tabular}{cccccccc}
\hline User & pri & qua & appe & co & ser & mail & time \\
\hline$U_{1}$ & & & & & & & \\
$U_{2}$ & & & & & & & \\
$\cdots$ & & & & & & & \\
\hline
\end{tabular}

In step 3 ,in accordance with equation (8)in the preceding part of the text, each user's trust degree is the minimum similarity value between $V_{i}$ and $L a_{i}$. This leaves this question as to how to compute the similarity value between $V_{i}$ and $L a_{i}$.

In step 4,we define a recursive function as follows : 


$$
f s(\boldsymbol{\theta}, \boldsymbol{\phi})=\left\{\begin{array}{lc}
r[i][j], & (\theta \in L a, \phi \in L a), \\
\min (f s(\operatorname{split}[k], \phi), & \text { if }(c 1), \\
1-r[i 1][j], & \text { if }(c 2), \\
\max (\operatorname{split} 1[k], \phi), & \text { if }(c 3), \\
\max (0, r[i 2][j]-r[i 3][j]), & \text { if }(c 4), \\
\min (1,1-r[i 3][j]+r[i 2][j]), & \text { if }(c 5), \\
\min (f s(\zeta, \phi), f s(\eta, \phi)), & \text { if }(c 6), \\
\max (f s(\zeta, \phi), f s(\eta, \phi)), & \text { if }(c 7) .
\end{array}\right.
$$

Within the above function,i is the index of $\theta$ in $\mathrm{La}, \mathrm{j}$ is the index of $\phi$ in $\mathrm{La}$; $\mathrm{c} 1$ is a condition that there is no logic operator $\neg, \vee$ in $\theta$, there are only $\wedge \mathrm{s}$ in $\theta$ expression, so the $\wedge$ split $\theta$ into $\mathrm{K}$ pieces of sub expression split[k], then $\mathrm{fs}(\theta, \phi)$ equal to the minimum of fs $(\mathrm{split}[\mathrm{k}], \phi)(\mathrm{k}=1, . . \mathrm{K})$; $\mathrm{c} 2$ represents that $\theta$ only has one operator $\neg$. It is written to the form as $\neg \varphi(\varphi \in \mathrm{La})$. i1 is the index of $\varphi$ in La; $\mathrm{c} 3$ is a condition that there is no logic operator $\neg, \wedge$ in $\theta$, there are only $\vee \mathrm{s}$ in $\theta$ expression, so the $\wedge$ split $\theta$ into $\mathrm{K}$ pieces of sub-expression split1[k], then $\operatorname{fs}(\theta, \phi)$ equal to the maximum of fs(split $1[\mathrm{k}], \phi)(\mathrm{k}=1, . . \mathrm{K})$; $\mathrm{c} 4$ points that that $\theta$ only has two operators $\neg$ and $\wedge$ . It is written to the form as $\neg \varphi \wedge \psi(\varphi, \psi \in \mathrm{La})$. i2 and $i 3$ are respectively the indexes of $\varphi, \psi$ in La; c5 is the condition that $\theta$ only has two operators $\neg$ and $\vee$. It is written to the form as $\neg \varphi \vee \psi(\varphi, \psi \in \mathrm{La})$,i2 and i3 are the same as above-mentioned ones;c6 is the condition that there are more than one operator $\wedge$ and more than one $\vee$, so $\theta$ can be split into two parts by the first operator, $\zeta \wedge \eta$ or $\zeta \vee \eta$. Here, $\min$ means to find a minimum value among its parameters, and max means to find maximum value among its parameters. This returning value from function fs $(\theta, \phi)$ which is the expression of $\theta_{s}$ is the similarity between $V_{i}$ and $L a_{i} . V_{i}$ and $L a_{i}$ correspond to two real parameters of fs function. $\theta$ gains value from $V_{i}$ expression and $L a_{i}$ pass the value to $\phi$. In step 5 . The output reputation value from step 3 and step 4 , a vague row vector $\mathrm{UT}(\mathrm{a} 1, \mathrm{a} 2, \mathrm{a} 3, \ldots)$, can be deducted according with equation (8). UT's size is equal to the length of La. a1 means the similarity degree between "very bad" and UT, a2 means the similarity degree between "bad" and UT, and so forth, and a5 the similarity degree between "very good" and UT. So here only know the similarity degree between $\mathrm{La}$ label and UT, but don't know the real value of UT. As we all know, a company have a wonderful image or an excellent reputation, the words "wonderful" and "excellent" indicate the level of goodness but not the specific number value. Because the fuzzy relation matrix points the similarity of every grade, we can normalize UT into one similarity degree. If we want to get the similarity degree between UT and "good" in La, the normalization equation, shown as equation 13 can be used.

normUT $=\frac{\sum_{i=1, \ldots, N} U T[i] * r[3][i]}{\sum_{i=1, \ldots, N} r[3][i]} \quad(N=$ La.length $)$

\section{Experiment analysis}

For testing and verifying the model and its algorithm,we suppose $\mathrm{La}$ has $\mathrm{N}$ grades. Because the similarity degree between every neighboring grade is $\theta_{s}$, the difference degree of it is $1-\theta_{s}$. then the greatest difference $\mathrm{N}\left(1-\theta_{s}\right)$ must meet $0 \leqslant(\mathrm{~N}-1)(1-$ $\left.\theta_{s}\right) \leqslant 1$; therefore, $1-\frac{1}{N-1} \leqslant \theta_{s} \leqslant 1$. In this experiment, $\mathrm{N}=5, \mathrm{La}=$ "VB,"B",'N","G","VG", so $1-\frac{1}{4} \leqslant$ $\theta_{s} \leqslant 1$, it is that $0.75 \leqslant \theta_{s} \leqslant 1$, set $\theta_{s}=0.75$. Company A's Ue data which consists of 30 days totalling 200 records comes from an online clothing store of www.taobao.com. Company B and C's data automatically comes from 4000 random records of 90 days by a program, then extracts 30 days data. Through the algorithm of section 5 , the curves of the similarity value between "good" and the reputation of company A,B and C are shown in Fig.4. 


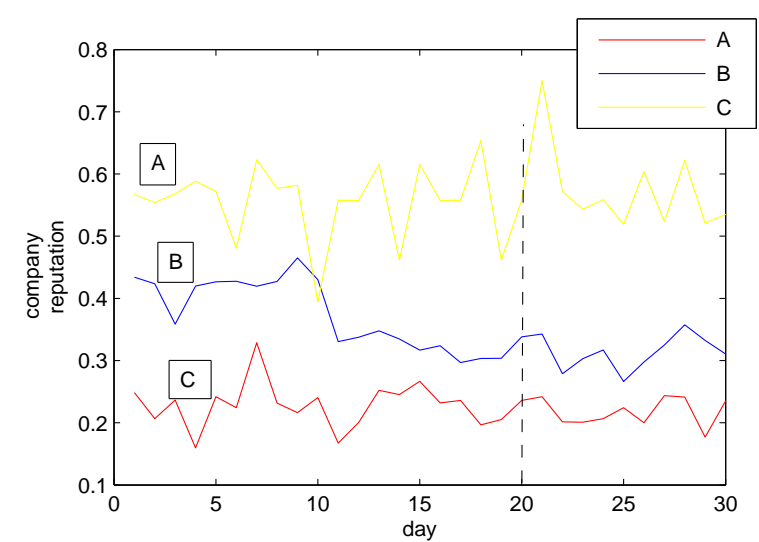

Fig. 4. Companies's Vague Reputation Comparison

This graph clearly indicates that during these 30 days, A's reputation is the best, B's is better than C's.On the 20th day, similar degree between A's reputation and "good" is 0.6. This means A has better prestige, $\mathrm{B}$ and C's are separately 0.25 and 0.35 , so $\mathrm{B}$ and $\mathrm{C}$ are not good enough.

Except for comparing the goodness degree of the reputation for different companies or stores, the itemized reputation of one company can also be computed. Fig.5 displays the reputation similar degree between the quality,the price,the appearance or the service of company A and "good". From the graphs, we can observe that the item's reputation is better and the service from 10 th day to 20 th day rises and falls. The quality still needs to improve. These curves not only can help customers to buy something but also benefit the boss to make their work better.
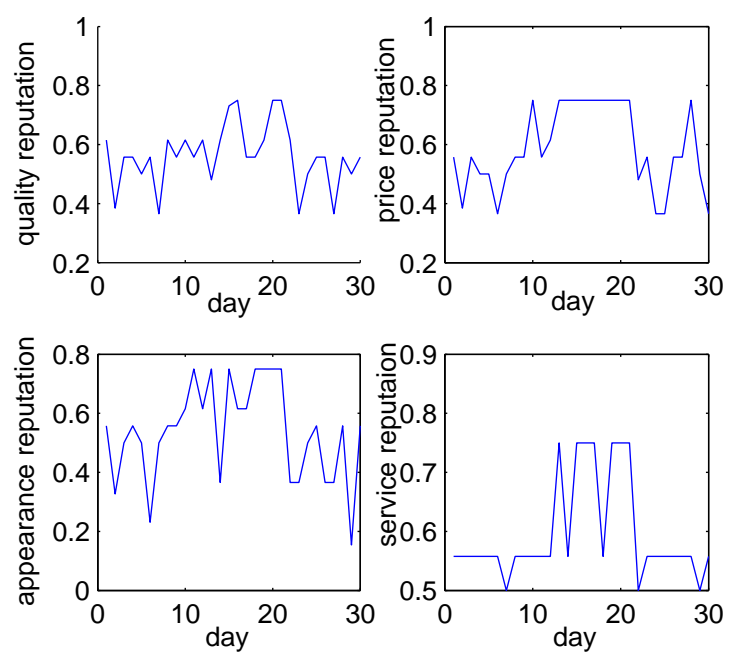

Fig. 5. Each Item Index Reputation Vague Value

\section{Application on E-commerce Behavioral Decision}

We propose a simple decision-making process for solving an e-commerce behavioral decision problem by means of the reputation evaluation approach presented in this paper. A new user is interested in a certain product of one company A where some users have been evaluated their transaction. Here for the description of convenience, we suppose four users $U_{1}, U_{2}, U_{3}, U_{4}$ who are evaluating the transaction for A of five alternatives $x_{1}, x_{2}, x_{3}, x_{4}, x_{5}$ using the linguistic words such as "very good, good, normal, bad, very bad" and are trying to obtain the history reputation $V_{r}$ about $\mathrm{A}$ and decide which side of $\mathrm{A}$ is the best alternative. These evaluations are assessed based on the linguistic label set $\mathrm{La}=\{\mathrm{VB}, \mathrm{B}, \mathrm{N}, \mathrm{G}$, $\mathrm{VG}\}$. We assume that the performance evaluations for all alternatives given by four users are given in the Table 2. In Table 2, the rows show a user's evaluating grades on each product or alternative of one seller. The columns show the user's grade of one product or alternative.

Table 2. User Evaluation Data.

\begin{tabular}{cccccc}
\hline User & $x_{1}$ & $x_{2}$ & $x_{3}$ & $x_{4}$ & $x_{5}$ \\
\hline$U_{1}$ & $\neg \mathrm{VB}$ & $\mathrm{N}$ & $\mathrm{B}$ & $\neg \mathrm{G}$ & $\neg \mathrm{G}$ \\
$U_{2}$ & $\mathrm{~N}$ & $\mathrm{~B}$ & $\mathrm{VB}$ & $\mathrm{B}$ & $\mathrm{N}$ \\
$U_{3}$ & $\mathrm{G}$ & $\neg \mathrm{VB}$ & $\mathrm{N}$ & $\mathrm{N}$ & $\mathrm{N}$ \\
$U_{4}$ & $\mathrm{G}$ & $\neg \mathrm{VG}$ & $\mathrm{B}$ & $\mathrm{B}$ & $\mathrm{B}$ \\
\hline
\end{tabular}

To obtain the collective the evaluation for each alternative and obtain the final decision, the semantic relation among linguistic labels La should be defined. Firstly, we define an order relation $<$ on La, that is $\mathrm{VB}<\mathrm{B}<\mathrm{N}<\mathrm{G}<\mathrm{VG}$. Secondly, we assume that the degree of semantic similarity $\theta_{s}$ between any two neighboring linguistic labels is 0.75 , according the order of $\{\mathrm{VB}, \mathrm{B}, \mathrm{N}, \mathrm{G}, \mathrm{VG}\}$, so from $\mathrm{R}$ (see matrix (14)) we get the element $r[i][j]$ in fuzzy relation $\mathrm{R}$ on La.

$$
\begin{aligned}
& \mathrm{R}= \\
& \left(\begin{array}{lllll}
1 & 0.75 & 0.5 & 0.25 & 0 \\
0.75 & 1 & 0.75 & 0.5 & 0.25 \\
0.5 & 0.75 & 1 & 0.75 & 0.5 \\
0.25 & 0.5 & 0.75 & 1 & 0.75 \\
0 & 0.25 & 0.5 & 0.75 & 0.1
\end{array}\right)
\end{aligned}
$$


It is clear that we can get two collects are represented by linguistic expressions; one is the collective evaluations of $x_{i}$ for all alternatives, that is the reputation for a certain product $P R_{i} . \mathrm{PR}\left(x_{1}\right)=\neg \mathrm{VB} \wedge \mathrm{N}$ $\wedge \mathrm{G} \wedge \mathrm{G} ; \mathrm{PR}\left(x_{2}\right)=\mathrm{N} \wedge \mathrm{B} \wedge \neg \mathrm{VB} \wedge \neg \mathrm{VG} ; \mathrm{PR}\left(x_{3}\right)$ $=\mathrm{B} \wedge \mathrm{VB} \wedge \mathrm{N} \wedge \mathrm{B} ; \mathrm{PR}\left(x_{4}\right)=\neg \mathrm{G} \wedge \mathrm{B} \wedge \mathrm{N} \wedge \mathrm{B}$ $; \mathrm{PR}\left(x_{5}\right)=\neg \mathrm{G} \wedge \mathrm{N} \wedge \mathrm{N} \wedge \mathrm{L}$.

Table 3. Reputation of Product $x_{i}$ to $\mathrm{X}$

\begin{tabular}{cc}
\hline Product & $\mathrm{PR}$ \\
\hline$x_{1}$ & $\neg \mathrm{VB} \wedge \mathrm{N} \wedge \mathrm{G} \wedge \mathrm{G}$ \\
$x_{2}$ & $\mathrm{~N} \wedge \mathrm{B} \wedge \neg \mathrm{VB} \wedge \neg \mathrm{VG}$ \\
$x_{3}$ & $\mathrm{~B} \wedge \mathrm{VB} \wedge \mathrm{N} \wedge \mathrm{B}$ \\
$x_{4}$ & $\neg \mathrm{G} \wedge \mathrm{B} \wedge \mathrm{N} \wedge \mathrm{B}$ \\
$x_{5}$ & $\neg \mathrm{G} \wedge \mathrm{N} \wedge \mathrm{N} \wedge \mathrm{L}$ \\
\hline
\end{tabular}

Now, from above Table 3 , we can compute the reputation $P R_{i}$ of each $x_{i}$ for enterprise A. The following Table 3 illustrates the degrees of similarities among $P R_{i}$ and the label in La.

Table 4. The Relations among $P R_{i}$ and The Labels in La.

\begin{tabular}{cccccc}
\hline Product & $\mathrm{VB}$ & $\mathrm{B}$ & $\mathrm{N}$ & $\mathrm{G}$ & $\mathrm{VG}$ \\
\hline$x_{1}$ & 0 & 0 & 0.25 & 0.5 & 0.5 \\
$x_{2}$ & 0 & 0 & 0.25 & 0 & 0 \\
$x_{3}$ & 0.5 & 0.75 & 0.5 & 0.25 & 0 \\
$x_{4}$ & 0.25 & 0.25 & 0 & 0 & 0 \\
$x_{5}$ & 0.25 & 0.25 & 0 & 0 & 0 \\
\hline
\end{tabular}

We select the linguistic labels having the maximum degrees of similarities as the performance evaluations for the alternatives. We can derive that linguistic label $\mathrm{G}$ or VG is the most appropriate evaluation for the alternative $\mathrm{x} 1, \mathrm{~N}$ is the most appropriate evaluation for the alternative $\mathrm{x} 2 \mathrm{~B}$ is the most appropriate evaluation for the alternative $\mathrm{x} 3, \mathrm{~B}$ or VB is the most appropriate evaluation for the alternatives $\times 4$ and $\times 5$. Hence, the final decision is that the performance of $\mathrm{x} 1$ is superior to others.

On the other hand, $U_{i}$ 's collective evaluations for all $x_{i}$ is the trust degree $U T_{i}$ for $U_{i}$ to A. $\mathrm{UT}\left(U_{1}\right)=$ $\neg \mathrm{VB} \wedge \mathrm{N} \wedge \mathrm{B} \wedge \neg \mathrm{G} \wedge \neg \mathrm{G} ; \mathrm{UT}\left(U_{2}\right)=\mathrm{N} \wedge \mathrm{B} \wedge$ $\mathrm{VB} \wedge \mathrm{B} \wedge \mathrm{N} ; \mathrm{UT}\left(U_{3}\right)=\mathrm{G} \wedge \neg \mathrm{VB} \wedge \mathrm{N} \wedge \mathrm{N} \wedge \mathrm{N}$; $\mathrm{UT}\left(U_{4}\right)=\mathrm{G} \wedge \neg \mathrm{VG} \wedge \mathrm{B} \wedge \mathrm{B} \wedge \mathrm{B}$. This computing is shown in following Table 5:

Table 5. User Trust Degree to $\mathrm{X}$

\begin{tabular}{cc}
\hline User & $\mathrm{UT}$ \\
\hline$U_{1}$ & $\neg \mathrm{VB} \wedge \mathrm{N} \wedge \mathrm{B} \wedge \neg \mathrm{G} \wedge \neg \mathrm{G}$ \\
$U_{2}$ & $\mathrm{~N} \wedge \mathrm{B} \wedge \mathrm{VB} \wedge \mathrm{B} \wedge \mathrm{N}$ \\
$U_{3}$ & $\mathrm{G} \wedge \neg \mathrm{VB} \wedge \mathrm{N} \wedge \mathrm{N} \wedge \mathrm{N}$ \\
$U_{4}$ & $\mathrm{G} \wedge \neg \mathrm{VG} \wedge \mathrm{B} \wedge \mathrm{B} \wedge \mathrm{B}$
\end{tabular}
ble 6 .

we can compute the degrees of similarities as Ta-

Table 6. The Relations among $U T_{i}$ s La.

\begin{tabular}{cccccc}
\hline User & $\mathrm{VB}$ & $\mathrm{B}$ & $\mathrm{N}$ & $\mathrm{G}$ & $\mathrm{VG}$ \\
\hline$U_{1}$ & 0 & 0 & 0 & 0 & 0.25 \\
$U_{2}$ & 0.5 & 0.75 & 0.5 & 0.25 & 0 \\
$U_{3}$ & 0 & 0 & 0.25 & 0.5 & 0.5 \\
$U_{4}$ & 0 & 0 & 0.25 & 0.25 & 0.25 \\
\hline
\end{tabular}

Then we can compute a history reputation $\left(V_{t}\right.$ in Fig3) according formula (9) (see table 6).h$\mathrm{REP}=\left\{\frac{1}{n} \sum_{i=1, \ldots, n} U T_{i}\right\}$ $=\left\{\frac{1}{4}((0+0.5+0+0),(0+0.75+0+0),(0+0.5+0.25+0.25)\right.$, $(0+0.25+0.5+0.25),(0.25+0+0.5+0.25)\}$

$=\{(0.125,0.185,0.25,0.25,0.25)\}$. so, therefor $\mathrm{h}-$ $\mathrm{REP}=\{(0.125,0.185,0.25,0.25,0.25)\}$. Based on the above vector h-REP. We normalize it into a "good" similarity degree called normUT by multiplying by the third row of $\mathrm{R}$ and dividing it by its sum(see. formula (13)). normUT $\doteq 0.2328$. If another company $\mathrm{B}$ 's normUT $=0.8$,then it can be stated that $\mathrm{B}$ is much better than $\mathrm{A}$.

\section{Conclusions}

A customer's evaluation content is often expressed by vague words, and a new model(RMBFR)is proposed to process fuzzy information in this paper. We show that RMBFR is very simple and satisfied some perfect performance because a fuzzy membership function did not need to be defined and therefore did not require vague information to be converted into numbers, or vice versa(see formulas (8) and (9)). Otherwise, reputation degree computing is defined and showed obeying the trust passing and trust combination principles. We give the model's algorithm and show the effective experiment. Finally, one application is illustrated and showed the 
method's practical usage. In formula(9),we equally treat every user's evaluation; we use $\frac{1}{n}$ to average the sum of users' trust value. In future work, we will consider the different user's weight.

\section{Acknowledgements}

This work supported by the National Natural Science Foundation of China (No. 61003254), the National Key Technology R\&D Program (No.2008BAH24B03), Zhejiang Provincial Natural Science Fund of China (No.Y1080130),Zhejiang Provincial Natural Science Fund of China (No.Y1101304) and "the Fundamental Research Funds for the Central Universities".

\section{References}

1. C. Elizabeth , D. Tharam, K. Farookh , "Trust and Reputation for Service-Oriented Environments: Technologies for Building Business Intelligence and Consumer Confidence," WILEY Press, Australia.58143(2008).

2. L. Li, "Reputation, Trust, and Rebates: How Online Auction Markets Can Improve Their Feedback Mechanisms",Journal of Economics \& Management Strategy, Volume 19, Issue 2, 303 C 331, (2010).

3. L. Martne, D. Ruan, F. Herrera, "Computing with Words in Decision support Systems: An overview on Models and Applications," International Journal of Computational Intelligence Systems, Vol.3, No. 4, 382-395(2010).

4. Y. Tang , J. Zheng, "Linguistic modelling based on semantic similarity relation among linguistic la- bels," Journal of Fuzzy Sets and Systems, 157, 16621673(2006).

5. A. JØsang ,SJ. Knapskog, "A metric for trusted system", Global IT Security, Wien:Australia Computer Society, 541-549(1998).

6. A. JØsang, "Trust-based decision making for electronic transactions". Proceeding of the 4th Nordic Workshop on Secure Computer System(NORDSEC'99), http://security.dstc.edu.au/staff/ajosang/paper.html.(1999).

7. A. JØsang, "Fuzzy Approach for the Evaluation of Trust and Reputation of Services," FUZZ-IEEE 2009, Korea, August 20-24, (2009).

8. L.A. Zadeh, "Fuzzy logic = computing with words," IEEE Trans , Fuzzy Systems, 4 (2) 103-111(1996).

9. G. Zhang, J. Kang, R. He, “ A review on information accessing systems based on fuzzy linguistic modelling "International Journal of Computational Intelligence Systems,v 3, n 4, p 420-437, October 2010; , (2010).

10. G. Zhang, J. Kang, R. He, " Towards a Trust Model with Uncertainty for e-Commerce Systems," Proceedings of the 2005 IEEE International Conference on eBusiness Engineering(ICEBE05), (2005).

11. A. Simone, J. Venkat P, “ Fuzzy Model for Trust Evaluation," FUZZ-IEEE 2009, Korea, August 20-24, (2009).

12. S. Zhang ,D. He, H. Andriy, "Fuzzy Approach for the Evaluation of Trust and Reputation of Services," Jounal of the southwest university(English), (1) 2006.

13. V. Buskens, " The social structure of trust," Social Networks, (20),265-298, (1998).

14. R. Marimon, J.P. Nicolini, and P. Teles, "Competition and reputation". Proceedings of the World Conference Econometric Society, Seattle, (2000).

15. D.B. Bromley, " Reputation, Image and Impression Management," John Wiley \& Sons,(1993). 